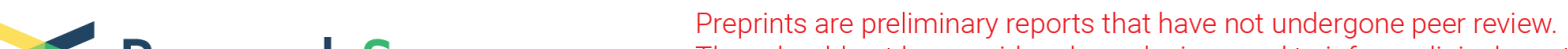 $\begin{array}{ll}\text { Research Square } & \text { They should not be considered conclusive, used to inform clinical practice, } \\ \text { or referenced by the media as validated information. }\end{array}$
}

\section{Non-steroidal anti-inflammatory drug use is determined by disease activity in axSpA and decreased by biologicals: a longitudinal analysis}

\section{Elif Durak Ediboglu}

Izmir Katip Celebi University Faculty of Medicine, Department of Internal Medicine, Division of Rheumatology

\section{Dilek Solmaz}

Izmir Katip Celebi University, Faculty of Medicine, Department of Internal Medicine, Division of Rheumatology

\section{Gökhan Kabadayı}

Izmir Katip Celebi University Faculty of Medicine Department of Internal Medicine Division of Rheumatology

\section{Sercan Gücenmez}

Izmir Katip Celebi University Faculty of Medicine Department of Internal Medicine Division of

Rheumatology

\section{Haluk Cinaklı}

Izmir Katip Celebi University Faculty of Medicine Department of Internal Medicine Division of Rheumatology

\section{Eda Otman Akad}

Izmir Katip Celebi University Faculty of Medicine Department of Internal Medicine Division of Rheumatology

\section{Mustafa Özmen}

Izmir Katip Celebi University Faculty of Medicine Department of Internal Medicine Division of Rheumatology

\section{Servet Akar ( $\sim$ servet.akar@gmail.com )}

Izmir Katip Celebi University Faculty of Medicine Department of Internal Medicine Division of Rheumatology https://orcid.org/0000-0002-3734-1242

\section{Research article}

Keywords: axial spondyloarthritis, non-steroidal anti-inflammatory drug use, ASAS-NSAID score

Posted Date: November 18th, 2021

DOI: https://doi.org/10.21203/rs.3.rs-1033541/v1 
License: (c) (i) This work is licensed under a Creative Commons Attribution 4.0 International License. Read Full License 


\section{Abstract \\ Objective}

To evaluate non-steroidal anti-inflammatory drug (NSAID) use and Assessment in Spondyloarthritis International Society (ASAS)-NSAID scores in patients with axial spondyloarhritis (axSpA) in a longitudinal study.

\section{Methods}

In total, 429 patients with axSpA (59\% male; $63.6 \%$ with AS) were included in this study. Data about disease activity, C-reactive protein (CRP) levels, and NSAID use and dosage were collected at 0, 12, 24, and 52 weeks retrospectively. The relationship with NSAID use /NSAID scores and other factors was tested using generalized estimating equations (GEE).

\section{Results}

At baseline (0 weeks), $92.8 \%$ of patients started biologic disease-modifying anti-rheumatic drugs (bDMARDs) and $82.1 \%$ were conventionally treated with NSAIDs. At baseline, the proportion $(p=0.03)$ and the median (IQR) ASAS-NSAID scores were higher in biologic treatment group [100 (50) vs 50 (83.4); $p<0.001]$. During follow-up, NSAID use and ASAS-NSAID scores decreased significantly in patients treated with bDMARDs $(p<0.001)$ and the reduction remained stable throughout the follow-up However, neither NSAID use $(p=0.06)$ nor ASAS-NSAID scores changed in conventionally treated patients $(p=0.15)$. In bDMARD-treated patients, ASDAS-CRP and BASFI scores were independent determinants for NSAID use, and BASDAl and patient global assessment (PGA) were determinants for NSAID dosage. There was no independent significant predictor for ASAS-NSAID scores; PGA was the only significant predictor for NSAID use in the conventional treatment group.

\section{Conclusion}

Concurrent biologic treatment was associated with low NSAID intake in patients with axSpA, and NSAID use was determined mainly by disease activity and partly by function during bDMARD treatment.

\section{Key Messages}

- NSAID use and dosage were significantly higher in patients with axSpA who need bDMARDs.

- NSAID use and ASAS-NSAID scores decreased significantly over time with biologic treatment.

- The disease activity measurements are associated with longitudinal NSAID use and/or dosage in axSpA. 


\section{Introduction}

Axial spondyloarthritis (axSpA) is a chronic, inflammatory rheumatic disease affecting the axial skeleton, which typically leads to inflammatory back pain. Axial spondyloarthritis is subdivided into two forms radiographic ( $\mathrm{r}$-axSpA) and non-radiographic axSpA (nr-axSpA) -based on the presence or absence of unequivocal radiographic changes in sacroiliac joints (1). The disease also causes peripheral arthritis, enthesitis, and dactylitis.

The treatment goals of axSpA are reducing symptoms, improving and maintaining spinal flexibility and normal posture, reducing functional limitations, maintaining the ability to work, and decreasing the extramusculoskeletal manifestations and complications associated with the disease (2).

The clinical efficacy of non-steroidal anti-inflammatory drugs (NSAIDs) on axSpA has been shown in numerous clinical trials (3-6), and NSAIDs are the first treatment option in patients with axial pain and/or stiffness $(2,7)$. NSAIDs improve back pain, spinal function, morning stiffness and also peripheral joint and entheseal pain. Moreover, there is also evidence suggesting that continuous or high NSAID intake may have an impact on slowing radiographic damage of the spine $(8,9)$. As a consequence, continuous and maximum tolerated doses of NSAIDs are recommended for active, symptomatic disease (9). However, NSAIDs may be associated with an increased risk of cardiovascular events, and gastrointestinal and renal toxicity $(10-13)$.

The Assessment in SpondyloArthritis international Society (ASAS)/European League Against Rheumatism (EULAR) management recommendations for axSpA advised biologic disease-modifying antirheumatic drugs (bDMARDs) for active axial disease despite at least two maximum doses of NSAIDs (2).

In daily clinical practice, tapering or withdrawing NSAIDs may be expected in patients who have begun taking bDMARDs. However, there are limited data concerning NSAIDs dosage and the determinants of NSAID use in patients with axSpA who have been treated with bDMARDs. To standardize the quantitation of NSAID use in patients with axSpA, the ASAS working group suggested using NSAID scores (14).

In a randomized placebo-controlled trial, baseline disease activity and ASAS-NSAID scores were similar between the groups; however, a significantly higher proportion of patients treated with etanercept vs. placebo reduced or stopped NSAID at week 8 (15). It was also shown in an observational study that treatment with tumor necrosis factor inhibitor (TNFi) was associated with a decrease in the proportion as well as the dosage of the NSAIDs in patients with axSpA in a real-life setting (16). Recently, another observational study revealed that longitudinal NSAID use was associated with disease activity, particularly in patients treated with TNFi (17).

Therefore, in the present study, we aimed to evaluate NSAID use longitudinally during a 52-week period in an observational cohort of patients with axSpA and to determine factors associated with NSAID intake in a real-life setting. 


\section{Materials And Methods}

\section{Study design and patient population}

This was a retrosprective observational study. All patients with axSpA were aged 18 years and over, according to the ASAS classification criteria for axSpA, patients from the SpA cohort of a tertiary referral hospital were included in the analysis (1).

Patients who had any contraindication for NSAID use such as pregnancy, lactation, and renal failure were excluded. This study was approved by the ethics committee of Izmir Katip Celebi University (approval number of 2020-0559).

In the present cohort, bDMARD treatment was started according to the ASAS/EULAR and American College of Rheumatology/Spondylitis Association of America/Spondyloarthritis Research and Treatment Network (ACR/SAA/SPARTAN) management recommendations and local guidelines $(2,7,18)$. NSAIDs were used based on ASAS/EULAR recommendations (7).

\section{Collected Data}

Baseline information regarding socio-demographic (including age, sex, education level, smoking status, body mass index) and disease-related characteristics were recorded. During follow-up (at 0, 12, 24, and 52 weeks), data about axSpA-related features [including the presence or the development of skeletal (peripheral arthritis, dactylitis, heel enthesitis)], extra-musculoskeletal manifestations [uveitis, psoriasis, inflammatory bowel disease (IBD)] in addition to disease activity and function measurements were collected.

The Bath Ankylosing Spondylitis Disease Activity Index (BASDAI), Bath Ankylosing Spondylitis Functional Disease Index (BASFI), serum C-reactive protein (CRP) levels, Ankylosing Spondylitis Disease Activity Score (ASDAS-CRP), and Patient Global Assessment (PGA) of disease activity were collected using a structured form. In each visit, NSAID intake, type, and the dosage and frequency of NSAIDs were also recorded to determine the ASAS-NSAID score.

The ASAS-NSAID score was calculated by using the following formula: (equivalent NSAID score) $\times$ (days of intake during the period of interest) $\times$ (days per week)/ (period of interest in days) as suggested by the ASAS working group (14).

\section{Statistical analysis}

All statistical analyses were performed using the Statistical Package of the Social Science (SPSS) software package version 13.0 (Chicago, IL). The distribution of continuous variables was investigated using visual (histograms, probability plots) and analytical methods (Kolmogorov-Smirnov). Most of the continuous variables were not distributed normally; therefore, values were presented as medians and 
interquartile range (IQR) for continuous and as percentages for categorical variables. The Mann-Whitney $U$ test was used to compare non-normally distributed variables between the groups. The Chi-square test or Fisher's exact test was used for the comparison of categorical data.

Friedman tests were conducted to test whether there was a significant change in the ASAS-NSAID scores and disease activity measurements due to violations of parametric test assumptions (non-normal distribution). The Wilcoxon test was performed to test the significance of pair-wise differences using Bonferroni correction to adjust for multiple comparisons.

NSAIDs use and scores over time were investigated using generalized estimating equations (GEE). The GEE procedure allows the analysis of repeated measurements in longitudinal studies. Traditional statistical techniques such as linear or logistic regression cannot be used in longitudinal studies because observations are not independent of each other and special techniques such as the GEE were developed to this end $(19,20)$. GEE is suitable for the evaluation of the longitudinal relationship with continuous and dichotomous outcome variables (dependent), and several time-dependent and independent covariates allow the use of unequal numbers of repeated measurement data, even if they deviate from normality (19).

In the first step of the present study, individual longitudinal interactions between ASAS-NSAID scores, demographic and clinical characteristics, and NSAID use were assessed and variables with $p$-values of $<0.10$ were included in regression models. Different multivariate longitudinal models were run, mainly differing in the disease activity measures.

All statistical tests were two-tailed and $p$-values of $<0.05$ were considered to be statistically significant.

\section{Results}

In total, 429 patients with axSpA (273 with $\mathrm{r}$ - and 156 with nr-axSpA) were included in the study. The median age was 40 (IQR: 18) years, $59 \%$ of patients were male and $61 \%$ were HLA-B27 positive. The median disease duration was 12 (IQR: 12) years. The baseline demographic and disease-related characteristics of the patients are summarized in Table 1.

Tablo 1. Baseline demographic and disease characteristics 


\begin{tabular}{|c|c|c|c|c|}
\hline & $\begin{array}{l}\text { All group } \\
(\mathrm{n}=429)\end{array}$ & $\begin{array}{l}\text { Biologic treatment } \\
(n=139)\end{array}$ & $\begin{array}{l}\text { Conventional } \\
\text { treatment }(n=290)\end{array}$ & $p$ \\
\hline Age, years median (IQR) & $40(18)$ & $39(19)$ & $41(17)$ & 0.49 \\
\hline Sex, male, n(\%) & $253(59)$ & $94(67.6)$ & $160(55)$ & 0.014 \\
\hline \multicolumn{5}{|l|}{ Diagnosis, n (\%) } \\
\hline r-AxSpA & $273(63.6)$ & 106 (76.3) & $166(57.4)$ & \\
\hline nr-AxSpA & $156(36.4)$ & $33(23.7)$ & $124(42.6)$ & $<0.001$ \\
\hline HLA-B27 positive, n (\%) & $\begin{array}{l}209 / 342 \\
(61.1)\end{array}$ & $64 / 95(67.4)$ & 146/248 (58.9) & 0.15 \\
\hline Smoking, ever, n (\%) & $\begin{array}{l}291 / 425 \\
(68.5)\end{array}$ & $89 / 135$ (65.9) & $202(69.7)$ & 0.44 \\
\hline $\begin{array}{l}\text { Duration of symptoms, } \\
\text { median yrs (IQR) }\end{array}$ & $12(12)$ & $14(11)$ & $11(12)$ & 0.004 \\
\hline $\begin{array}{l}\text { Time since diagnosis, } \\
\text { median yrs (IQR) }\end{array}$ & $5(7)$ & $7(8)$ & $4(5)$ & $<0.001$ \\
\hline \multicolumn{5}{|c|}{ The presence of extraspinal/ extra-musculoskeletal manifestations history } \\
\hline Periferal arthritis, n (\%) & $\begin{array}{l}127 / 407 \\
(31.2)\end{array}$ & $60 / 123(48.8)$ & $67 / 285(23.5)$ & $<0.001$ \\
\hline Enthesitis, n (\%) & $\begin{array}{l}108 / 377 \\
(48)\end{array}$ & $52 / 99$ (52.5) & $129 / 279(46.2)$ & 0.30 \\
\hline Dactylitis, n (\%) & $\begin{array}{l}14 / 414 \\
(3.4)\end{array}$ & $7 / 126(5.6)$ & $7 / 288(2.4)$ & 0.11 \\
\hline Hip involvement , n (\%) & $\begin{array}{l}51 / 366 \\
(14.2)\end{array}$ & 18/95 (18.9) & $33 / 271(12.2)$ & 0.10 \\
\hline Anterior uveitis, $\mathrm{n}(\%)$ & $\begin{array}{l}45 / 413 \\
(10.9)\end{array}$ & $17 / 126(13.5)$ & $28 / 287(9.8)$ & 0.26 \\
\hline Psoriasis, n (\%) & $\begin{array}{l}17 / 411 \\
(4.1)\end{array}$ & $5 / 126(4)$ & $12 / 285(4.2)$ & 0.91 \\
\hline $\begin{array}{l}\text { İnflamatory bowel disease, } \\
n(\%)\end{array}$ & $\begin{array}{l}10 / 369 \\
(2.7)\end{array}$ & $5 / 96(5.2)$ & $5 / 273(1.8)$ & 0.08 \\
\hline \multicolumn{5}{|l|}{ Disease activity, median (IQR) } \\
\hline BASDAI (0 to 10$)$ & $4(3)$ & $4.9(2.8)$ & $3.6(2.8)$ & $<0.001$ \\
\hline BASFI (0 to 10$)$ & $2.9(4.2)$ & $4.4(3.7)$ & $2.2(3.9)$ & $<0.001$ \\
\hline BASMI (0 to 10) & $2(3)$ & $3(3)$ & $2(2)$ & 0.009 \\
\hline ASDAS-CRP & $2.8(1.5)$ & $3.5(1.4)$ & $2.4(1.4)$ & $<0.001$ \\
\hline
\end{tabular}




\begin{tabular}{|lllll|}
\hline CRP $(\mathrm{mg} / \mathrm{dl})$ & $1(8)$ & $7(15.8)$ & $1(0.5)$ & $<0.001$ \\
\hline PGA of disease activity & & & $40(40)$ & $<0.001$ \\
$(0$ to 100$)$ & $40(40)$ & $60(30)$ & & \\
\hline Concomitant medications & & & $238(82.1)$ & 0.003 \\
\hline Current NSAID use n,\% & $367(85.5)$ & $129(92.8)$ & $67 / 274(25)$ & 0.32 \\
\hline Current cDMARD use $\mathrm{n}, \%$ & $93 / 405(23)$ & $26 / 131(20)$ & \\
\hline
\end{tabular}

AxSpA: Axial spondyloarthritis, r-axSpA: radiographic axSpA, nr-axSpA: non-radiographic axSpA, AS: Ankylosing Spondylitis, HLA-B27: Human leucocyte antigen B27, BASDAl: Bath AS Disease Activity Index, BASFI: Bath AS Functional Disease Index, BASMI: Bath AS Metrology Index, CRP: serum C reactive protein , ASDAS-CRP: Ankylosing Spondylitis Disease Activity Score-CRP, PGA: patient global assessment, NSAID: non-steroidal anti-inflammatory drug, CDMARD: conventional disease-modifying antirheumatic drug

In the present study, 139 patients with axSpA were started on bDMARDs and 290 were treated conventionally. There was a higher proportion of males and patients with r-axSpA among the biologic treatment group. Furthermore, symptom duration and time from diagnosis were longer and, as expected, disease activity was higher in patients started on bDMARDs.

At baseline, 129 out of 139 (92.8\%) patients started biologic treatment [92.1\% of patients on TNFi (infliximab, adalimumab, etanercept, certolizumab, golimumab) and $7.9 \%$ on secukinumab] and 238 (82.1\%) patients were treated conventionally using NSAIDs, and the median ASAS-NSAID scores were 100 (IQR: 50) and 50 (IQR: 83.4), respectively. Both the proportion of NSAID use (figure 1$)(p=0.03)$ and the median ASAS-NSAID scores (supplementary figure 1) were statistically higher in the bDMARD-treated group $(p<0.001)$.

During the follow-up period, the proportion of NSAID use (figure 1) and, in particular, ASAS-NSAID scores (supplementary figure 1) decreased rapidly (as early as 12 weeks) and significantly in patients treated with biologic drugs; the reduction remained stable throughout follow-up $(p<0.001)$. However, neither NSAID use $(p=0.06)$ nor ASAS-NSAID scores changed in conventionally treated patients $(p=0.15)$.

All disease activity measurements (BASDAl, BASFI, ASDAS-CRP, serum CRP levels, PGA of disease activity) decreased significantly in bDMARD-started patients in week $12(p<0.001)$ and the measurements were found to be stable (supplementary figure 2-6) throughout follow-up. However, disease activity did not change in conventionally treated patients during the follow-up period (supplementary figure 2-6).

\section{NSAID use as a continuous variable estimated using ASAS- NSAID scores}


In the entire axSpA cohort, longitudinal univariate analysis revealed that ASAS-NSAID scores were significantly associated with treatment (B:15.010, 95\% Cl: [8.879- 21.142]; $\mathrm{p}<0.001$ for bDMARD), BASDAI (B:6.807, 95\%Cl: [5.514- 8.099]; $p<0.001)$, BASFI (B:5.248, 95\%Cl: [4.064- 6.431]; $p<0.001), P G A$ of disease activity scores (B:0.468, 95\% Cl: [0.350- 0.585]; $p<0.001$ ), and serum CRP levels (B:0.153, 95\%Cl: [-0.0200.326]; $p=0.082)$ in the multivariate analysis, BASDAI (B:2.885, 95\%Cl: [0.441- 5.330]; $p=0.021), P G A$ of disease activity scores (B:0.365, 95\% Cl: [0.188- 0.542]; $p<0.001)$, and treatment type $(\mathrm{B}: 14.515,95 \% \mathrm{Cl}$ : [6.620- 22.409]; $p<0.001$ for bDMARD) were independent determinants of ASAS-NSAID scores in patients with axSpA.

We repeated the longitudinal analysis in the conventional and bDMARD-treated groups separately after whole-group analysis. Univariate longitudinal analysis in the biologic treated group revealed that ASASNSAID scores were significantly associated with ASDAS-CRP (B:8.104, 95\%Cl: [0.621- 15.586]; $p=0.034)$, BASDAI (B:10.948, 95\%Cl: [9.302-12.585]; $p<0.001)$, BASFI (B:7.716, 95\%Cl: [6.185-9.247]; $p<0.001)$ and PGA of disease activity (B:0.813, 95\%Cl: [0.664-0.961]; $p<0.001)$ and serum CRP levels $(\mathrm{B}: 0.250,95 \% \mathrm{Cl}$ : [0.046- 0.453]; $p=0.016)$. Therefore, we established two multivariable models to assess the associated factors/covariates with ASAS-NSAID scores over time (one with ASDAS and the other BASDAI+CRP as disease activity measures) and showed that BASDAI and PGA of disease activity were independent determinants of ASAS-NSAID scores in patients who were biologically treated (Table 2).

Table 2

The factors associated with ASAS-NSAID scores in biologic treated patients

\begin{tabular}{|c|c|c|c|c|c|c|}
\hline & & Model 1 & & & Model 2 & \\
\hline & B & $95 \% \mathrm{Cl}$ & $\mathrm{p}$ & B & $95 \% \mathrm{Cl}$ & $\mathrm{P}$ \\
\hline BASFI & -0.55 & $-4.384-4.275$ & 0.980 & 0.943 & $-3.795-5.682$ & 0.696 \\
\hline BASMI & 0.951 & $-1.511-3.413$ & 0.449 & -0.098 & $-2.430-2.234$ & 0.934 \\
\hline PGA & 0.535 & $0.210-0.860$ & 0.001 & 0.759 & $0.432-1.086$ & $<0.001$ \\
\hline CRP & -0.068 & $-0.220-0.084$ & 0.380 & & & \\
\hline BASDAI & 5.718 & $2.487-8.949$ & 0.001 & & & \\
\hline ASDAS-CRP & & & & 1.771 & $-6.571-10.113$ & 0.677 \\
\hline
\end{tabular}

Although univariate longitudinal analysis revealed that ASAS-NSAID scores were associated with BASDAI (B:2.812, 95\%Cl: [1.095-4.529]; $p<0.001), \mathrm{BASFI}$ (B:2.941, 95\%Cl: [1.358-4.523]; $p<0.001)$, PGA of disease activity scores (B:0.296, 95\% Cl: [0.145-0.448]; $p<0.001)$, there was no independent significant predictor for ASAS-NSAID scores in the conventional treatment group in multivariate analysis for smoking (B:-8.391, 
95\%Cl: [-16.851- 0.069]; $p=0.052)$, education (B:-0.936, 95\%Cl: [-1.823 to -0.048$] ; p=0.039)$ and history of psoriasis (B:20.154, 95\%Cl: [-3.04 to -43.348]; $\mathrm{p}=0.089)$ (Table 3).

Table 3

The factors associated with ASAS-NSAID scores in conventionally treated patients

\begin{tabular}{|llll|}
\hline & B & $95 \%$ Cl & p \\
\hline BASDAI & 1.365 & $-2.305-5.035$ & 0.466 \\
\hline BASFI & 1.007 & $-2.166-4.181$ & 0.534 \\
BASMI & 0.275 & $-2.560-3.110$ & 0.849 \\
\hline PGA & 0.229 & $-0.016-0.474$ & 0.067 \\
\hline Smoking (never) & $-6,894$ & $-17,320-3,532$ & 0.195 \\
\hline Education (years) & -0.895 & $-2.166-0.377$ & 0.168 \\
\hline Psoriasis (no) & 11,632 & $-17.450-40.714$ & 0.433 \\
\hline $\begin{array}{l}\text { BASDAl: Bath AS Disease Activity Index, BASFI: Bath AS Functional Disease Index, BASMI: Bath AS } \\
\text { Disease Activity Index, AS: Ankylosing Spondylitis, PGA: patient global assessment of disease activity }\end{array}$ \\
\hline
\end{tabular}

\section{NSAID use as a nominal variable}

In additions to ASAS-NSAID scores, we analyzed NSAID use as a dichotomous variable in univariate and multivariate longitudinal analysis in the entire axSpA group as well as in separate treatment groups. Univariate longitudinal analysis revealed that in all patients, NSAID use was significantly associated with treatment type (B:0.808, 95\%Cl: [0.456-1.161]; $\mathrm{p}<0.001$ for bDMARD), BASDAI (B:0.357, 95\%Cl: [0.2470.467]; p<0.001), BASFI (B:0.254, 95\%Cl: [0.160- 0.347], p<0.001), ASDAS-CRP (B:0.608, 95\%Cl: [0.3870.829]; $p<0.001), P G A$ of disease activity scores (B:0.024, 95\%Cl: [0.014- 0.034 ]; $p<0.001)$, and history of extra-musculoskeletal manifestations (B:0.518, 95\%Cl: [0.55- 0.981]; $p=0.028)$. In the multivariable model, assessing the associated factors/covariates with NSAID use over time showed that treatment type (B:0.655, 95\%Cl: [0.126- 1.185]; $p=0.015$ for bDMARD), BASDAI (B:0.259, 95\%Cl: [0.063- 0.455]; $p=0.010)$, BASFI (B:0.273, 95\%Cl: [0.160- 0.386]; $p<0.001)$ and PGA of disease activity (B:0.014, 95\%Cl: [0.0020.027 ]; $p=0.026$ ) scores were independent determinants of NSAID use in patients with axSpA.

Then, we repeated the univariate longitudinal analysis for the determinants of NSAID use in each treatment group and found that in the biologically treated group, NSAID use was significantly associated with BASDAI (B:0.610, 95\%Cl: [0.439- 0.781]; p<0.001), BASFI (B:0.406, 95\%Cl: [0.269- 0.544]; $p<0.001)$, ASDAS-CRP (B:0.791, 95\%Cl: [0.507-1.075]; $p<0.001)$ and PGA of disease activity scores (B:0.037, $95 \% \mathrm{Cl}$ : [0.021-0.053]; $p<0.001)$. Therefore, we again established two multivariable models to assess the associated factors/covariates with NSAID use over time (one with BASDAI and the other ASDAS-CRP as disease activity measures) and showed that ASDAS-CRP and BASFI were independent determinants of NSAID use in patients with axSpA treated with bDMARDs (Table 4). 
Table 4

The factors associated with NSAID use in biologic treated patients

\begin{tabular}{|c|c|c|c|c|c|c|}
\hline & \multicolumn{3}{|c|}{ Model 1} & \multicolumn{3}{|c|}{ Model 2} \\
\hline & B & $95 \% \mathrm{Cl}$ & $\mathrm{p}$ & B & $95 \% \mathrm{Cl}$ & $P$ \\
\hline BASDAI & 0.247 & $-0.039-0.532$ & 0.090 & & & \\
\hline PGA & 0.020 & $-0.002-0.042$ & 0.072 & & & \\
\hline BASFi & 0.032 & $-0.201-0.265$ & 0.789 & 0.229 & $0.053-0.404$ & 0.011 \\
\hline ASDAS-CRP & & & & 0.527 & $0.174-0.880$ & 0.03 \\
\hline
\end{tabular}

Although univariate longitudinal analysis revealed that NSAID use was associated with age (B:-0.022, 95\%Cl: [-0.043 to -0.001]; $p=0.041$ ), symptom duration (B:-0.024, 95\%Cl: [-0.043 to -0.001$] ; p=0.014), P G A$ of disease activity (B:0.016, 95\% Cl: [0.004- 0.029]; $\mathrm{p}=0.013)$, and history of extra-musculoskeletal manifestations ( $\mathrm{B}: 0.701,95 \% \mathrm{Cl}$ : [0.108-1.294]; $\mathrm{p}=0.021)$ in the conventional treatment group, multivariate analysis showed that PGA of disease activity was the only significant predictor for NSAID use in this group of patients with axSpA (Table 5).

Table 5

The factors associated with NSAID use in conventionally treated patients

\begin{tabular}{|lccc|}
\hline & B & $95 \%$ Cl & p \\
\hline PGA & 0.017 & 0.0040 .031 & 0.011 \\
\hline Age & -0.016 & -0.0520 .020 & 0.391 \\
\hline Symptom duration (years) & -0.023 & -0.0550 .008 & 0.150 \\
\hline History of EMM & 0.419 & -0.3981 .236 & 0.315 \\
\hline PGA: patient global assessment of disease activity, EMM; extra-musculoskeletal manifestations \\
\hline
\end{tabular}

\section{Discussion}

The results of this long-term follow-up study showed that NSAID use in patients with axSpA was determined mainly by disease activity and partly by function. Almost all of the activity measurements used in daily clinical practice, such as BASDAI, PGA of disease activity, and serum CRP levels were found to be associated with longitudinal NSAID use in the present cohort. However, the disease subtype had no impact on both NSAID use and dosage in patients with axSpA. In our study groups, biologic treatment was associated with both clinical improvement and a decrease in NSAID use and dosage. Using GEE 
analysis, we also showed that in biologic treated patients, NSAID use was determined mainly by disease activity, irrespective of treatment type during the follow-up. There are limited data evaluating the determinants of longitudinal NSAID use in patients with axSpA (15-17).

In our study, at the baseline visit, both the proportion of NSAID use and the median ASAS-NSAID scores were statistically higher in the biologic treated patients with axSpA. However, both NSAID use and dosage decreased significantly only in the advanced treatment group. Similar to our findings, NSAID use and dosage were found to be decreased in follow-up in comparison with the baseline visit in patients with axSpA treated with TNFi in the Groningen Leeuwarden Ankylosing Spondylitis (GLAS) cohort.

Nevertheless, in contrast to our findings, the authors also reported that baseline NSAID use was similar in the TNFi and conventional treatment groups (79\% vs $74 \% ; p=0.131)(17)$. The reason for the high NSAID use in both treatment groups was not clear in this study because baseline disease activity was higher in the patients who were put on TNFi treatment (17).

NSAIDs are the first choice of treatment in patients with axial pain and/or stiffness and biologic agents should be considered in patients who have high disease activity despite the maximum tolerable dose of NSAIDs. In the GLAS cohort, the proportion of NSAID use was lower in the TNFi treatment group than in our study; this result might be related to the requirement of TNFi treatment for some different involvements in patients in the GLAS cohort; the higher conventional DMARD use in the patients with TNFi treatment supports this hypothesis. In both the Devenir des Spondylarthropathies Indifférenciées Récentes (DESIR) cohort and SPARSE study, NSAID use was similar to our patients with axSpA and higher than in the GLAS cohort (15-17).

In our study, we analyzed the entire axSpA patient group as well as the biologically and conventionally treated groups separately to understand the determinants of NSAID use and dosage longitudinally. In our axSpA cohort, longitudinal multivariate analysis revealed that, besides disease activity, therapy with bDMARDs was an independent predictor of NSAID use as well as dosage, suggesting a NSAID-sparing effect of bDMARDs in patients with axSpA. In the GLAS cohort, the authors also revealed to NSAID use was significantly related with disease activity (17). However, they analyzed conventional and bDMARD groups separately and did not evaluate whole group of patients. Also, they only used ASDAS-CRP, BASDAl, and serum CRP levels as disease activity measurements. In our study, we additionally analyzed function and PGA of disease activity for patient-reported outcomes. Furthermore, when we assessed patients in two treatment groups (conventional and biologic), we found that NSAID use was related to BASDAI, ASDAS-CRP, BASFI, and PGA of disease activity scores in biologically treated patients. By contrast, PGA of disease activity was the only determinant of NSAID use in the conventional treatment group. In addition to ASAS-NSAID scores, we analyzed NSAID use as a nominal variable. However, we believe that ASAS-NSAID scores could be a more reliable and objective marker for NSAID use in patients with axSpA because they are calculated using equivalent NSAID scores, dosage, and the percentage of days with NSAID use in a defined period. As shown in our study, in the SPARSE study, which evaluated the NSAID-sparing effect of TNFi agents in patients with axSpA, the primary endpoint was ASAS-NSAID scores to examine this effect and the authors showed the importance of ASAS-NSAID scores (15). 
The results of the present study provide additional evidence regarding the substantial efficacy of TNFi in patients with axSpA because all disease activity measures decreased significantly after as little as 12 weeks of follow-up. Furthermore, in our biologic treatment group, the proportion of patients using NSAIDs and median ASAS-NSAID scores decreased in 12 weeks and remained stable at the 24 and 52-weeks follow-up visits. The authors of the GLAS cohort study showed that ASAS-NSAID scores significantly decreased with TNFi therapy in 6-12 weeks (17). In the SPARSE study, NSAID use was reduced or stopped at week 8 in patients treated with etanercept (15). The percentage of patients with axSpA, in whom NSAID scores decreased or NSAID treatment was discontinued, was higher among the TNFi treatment group, also in the DESIR cohort (16).

Our study has some limitations. First, it was a retrospective study, inevitably resulting in missing data in the follow-up period. Second, we did not evaluate the different treatment groups (TNFi agents and secukinumab in addition to COX-2 inhibitors and traditional NSAIDs) as a sub-analysis due to the low number of patients.

The main strengths of the study were the patient populations and sample size because patients with both $r$ - and nr-axSpA were included in the study. This is the first study to show that in addition to biologic treatment, disease activity might affect NSAID use and dosage in patients with both $r$ - and nr-axSpA. Additionally, we used GEE for longitudinal analysis, which is suitable for the evaluation of the relationship with continuous as well as dichotomous outcome variables and several time dependent and independent covariates.

\section{Conclusion}

This is the first study to evaluate the relationship between NSAID use and disease activity of all subtypes of axSpA in a longitudinal analysis. Our results showed that NSAID use and dosage were significantly higher in patients with axSpA who were indicated bDMARD treatment. NSAID use decreased significantly over time with biologic treatment (as soon as 12 weeks) and independently determined by disease activity. However, it was stable in conventionally treated patients with axSpA. Calculating ASAS-NSAID scores may be a more appropriate outcome than nominal NSAID use to investigate NSAID use in patients with axSpA.

\section{Abbreviations}

AxSpA

Axial spondyloarthritis

ASAS

The Assessment in SpondyloArthritis international Society ASDAS-CRP

Ankylosing Spondylitis Disease Activity Score

BASDAI

Page 13/18 
Bath Ankylosing Spondylitis Disease Activity Index

BASFI

Bath Ankylosing Spondylitis Functional Disease Index

bDMARDs

biologic disease-modifying antirheumatic drugs

CRP

C-reactive protein

EULAR

European League Against Rheumatism

GEE

generalized estimating equations

IBD

inflammatory bowel disease

IQR

interquartile range

NSAIDs

non-steroidal anti-inflammatory drugs

nr-AxSpA

non-radiographic Axial spondyloarthritis

PGA

Patient Global Assessment

r-AxSpA

Radiographic Axial spondyloarthritis

TNFi

tumor necrosis factor inhibitor

\section{Declarations}

Ethical Approval and consent to participate: This study was approved by the ethics committee of Izmir Katip Celebi University (approval number of 2020-0559)

Consent for publication: Not applicable

Availability of supporting data: Not applicable

Funding: No specific funding was received from any bodies in the public, commercial or not-for-profit sectors to carry out the work described in this article.

Conflict of interests: No conflict of interests

Authors' contributions: EDE, DS and SA contributed to the conception and design of this study. GK, SG, $\mathrm{HC}$ and EOA participated in data collection. EDE, SA, DS and MO contributed to the data analysis. All 
authors contributed to the data interpretation, reviewed the article and approved the final draft for submission.

\section{Acknowledgements: None}

\section{Authors' information:}

Elif Durak Ediboğlu, MD, Izmir Katip Celebi University, Faculty of Medicine, Department of Internal Medicine, Division of Rheumatology, Izmir, TURKEY

Dilek Solmaz, MD, Assoc Prof, Izmir Katip Celebi University, Faculty of Medicine, Department of Internal Medicine, Division of Rheumatology, Izmir, TURKEY

Gökhan Kabadayı, MD, Izmir Katip Celebi University, Faculty of Medicine, Department of Internal Medicine, Division of Rheumatology, Izmir, TURKEY

Sercan Gücenmez, MD, Izmir Katip Celebi University, Faculty of Medicine, Department of Internal Medicine, Division of Rheumatology, Izmir, TURKEY

Haluk Cinaklı, MD, Izmir Katip Celebi University, Faculty of Medicine, Department of Internal Medicine, Division of Rheumatology, Izmir, TURKEY

Eda Otman Akat, MD, Izmir Katip Celebi University, Faculty of Medicine, Department of Internal Medicine, Division of Rheumatology, Izmir, TURKEY

Mustafa Özmen, MD, Assoc Prof, Izmir Katip Celebi University, Faculty of Medicine, Department of Internal Medicine, Division of Rheumatology, Izmir, TURKEY

Servet Akar, MD, Prof, Izmir Katip Celebi University, Faculty of Medicine, Department of Internal Medicine, Division of Rheumatology, Izmir, TURKEY

Corresponding author: Servet Akar

Izmir Katip Celebi University, Faculty of Medicine, Department of Internal Medicine, Division of Rheumatology

Basın sitesi street, 35050, Izmir, Turkey

ORCID ID: 0000-0002-3734-1242

Telephone number: 5055252005

servet.akar@gmail.com 


\section{References}

1.Rudwaleit M, van der Heidje D, Landewé R, Listing J, Akkoc N, Brandt J, et al. The development of the Assessment of SpondyloArthritis International Society classification criteria for axial spondyloarthritis (part II): validation and final selection. Ann Rheum Dis 2009; 68:777-83.

2. van der Heijde D, Ramiro S, Landewé R, Baraliakos X, Van den Bosch F, Sepriano A, et al. 2016 update of the ASAS-EULAR management recommendations for axial spondyloarthritis. Ann Rheum Dis. 2017;76:978.

3. Barkhuizen A, Steinfeld S, Robbins J, West C, Coombs J, Zwillich S. Celecoxib is eficious and well tolerated in treating signs and symptoms of ankylosing spondylitis. Journal of Rheumatology 2006; 33(9):1805-12.

4. Van der Heijde D, Baraf HS, Ramos-Remus C, et al. Evaluation of the efficacy of etoricoxib in ankylosing spondylitis: results of a fifty-two-week, randomized, controlled study. Arthritis Rheum 2005; 52:1205-15

5. Dougados M, Gueguen A, Nakache JP, Velicitat P, Veys EM, Zeidler H, et al. Ankylosing spondylitis: what is the optimum duration of a clinical study? A one year versus a 6 weeks non-steroidal anti-inflammatory drug trial. Rheumatology 1999; 38(3):235-44

6. Khan MA. A double blind comparison of diclofenac and indomethacin in the treatment of ankylosing spondylitis. Journal of Rheumatology 1987; 14(1):118-23

7. Ward MM, Deodhar A, Gensler LS, Dubreuil M, Yu D, Khan MA' et al. 2019 Update of the American College of Rheumatology/Spondylitis Association of America/Spondyloarthritis Research and Treatment Network Recommendations for the Treatment of Ankylosing Spondylitis and Nonradiographic Axial Spondyloarthritis. Arthritis Rheumatol. 2019; 71:1599

8.Kroon $F$, Landewé $R$, Dougados $M$, van der Heijde D. Continuous NSAID use reverts the effects of inflammation on radiographic progression in patients with ankylosing spondylitis. Ann Rheum Dis. 2012 Oct;71(10):1623.

9. Poddubnyy D, Rudwaleit M, Haibel H, Listing J, Märker-Hermann E, Zeidler H, et al. Effect of nonsteroidal anti-inflammatory drugs on radiographic spinal progression in patients with axial spondyloarthritis: results from the German Spondyloarthritis Inception Cohort. Ann Rheum Dis. 2012 Oct; 71(10):1616-22.

10. Gabriel SE, Jaakkimainen L, Bombardier C. Risk for serious gastrointestinal complications related to use of nonsteroidal anti-inflammatory drugs. A meta-analysis. Annals of Internal Medicine 1991;115(10):787-96. 
11. Stalnikowicz R, Rachmilewitz D. NSAID-induced gastroduodenal damage: is prevention needed? A review and meta-analysis. Journal of Clinical Gastroenterology 1993; 17(3):238-43.

12.Trelle S, Reichenbach S, Wandel S, Hildebrand P, Tschannen B, Villiger PM, et al. Cardiovascular safety of non-steroidal anti-inflammatory drugs: network meta-analysis. BMJ 2011;342:c7086.

13.Murray MD, Brater DC. Renal toxicity of the nonsteroidal anti-inflammatory drugs. Annual Review of Pharmacology and Toxicology 1993; 33:435-65

14.Dougados M, Simon P, Braun J, Burgos-Vargas R, Maksymowych WP, Sieper J, et al. ASAS recommendations for collecting, analysing and reporting NSAID intake in clinical trials/ epidemiological studies in axial spondyloarthritis. Ann Rheum Dis 2011; 70:249-51.

15. Dougados M, Wood E, Combe B, Schaeverbeke T, Miceli-Richard C, Berenbaum F, et al. Evaluation of the nonsteroidal anti-inflammatory drug-sparing effect of etanercept in axial spondyloarthritis: results of the multicenter, randomized, double-blind, placebo-controlled SPARSE study. Arthritis Res Ther 2014 Nov 27; 16(6):481-014-0481-5.

16. Molto A, Granger B, Wendling D, Breban M, Dougados M, Gossec L. Brief Report: Nonsteroidal Antiinflammatory Drug-Sparing Effect of Tumor Necrosis Factor Inhibitors in Early Axial Spondyloarthritis: Results From the DESIR Cohort. Arthritis Rheumatol 2015 Sep; 67(9):2363 \pm 2368.

17. Carbo MJG, Spoorenberg A, Maas F, Brouwer E, Bos R, Bootsma H, et al. Ankylosing spondylitis disease activity score is related to NSAID use, especially in patients treated with TNF-a inhibitors. PLoS ONE 2018 Apr 24; 13(4): e0196281.

18. https://www.teb.org.tr/content/75/SUT

19. Wang M, Generalized Estimating Equations in Longitudinal Data Analysis: A Review and Recent Developments. Hindawi Publishing Corporation, Advances in Statistics Volume 2014, Article ID 303728, 11 pages.

20. Twisk JWR, Longitudinal data analysis. A comparison between generalized estimating equations and random coefficient analysis. European Journal of Epidemiology 19: 769-776, 2004.

\section{Figures}




\section{NSAID usage in groups}

100

90

80

70

60

50

40

30

20

10

0
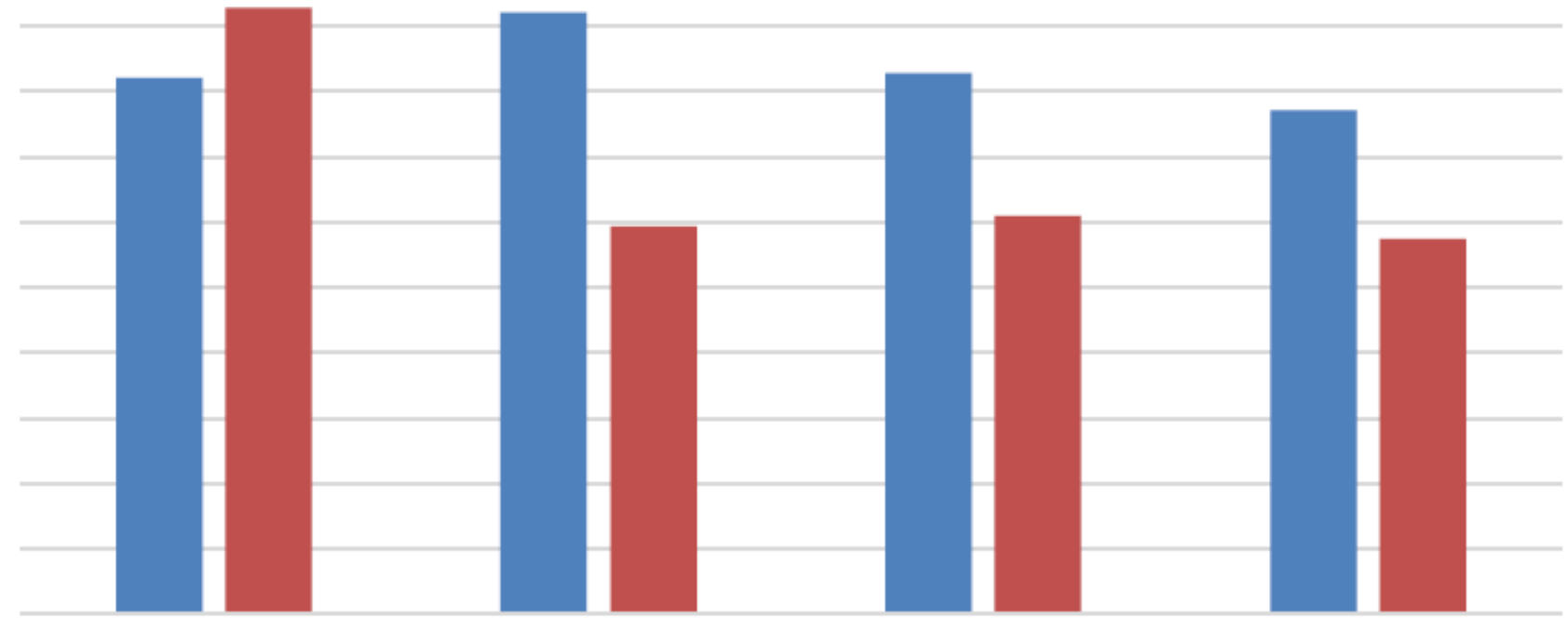

Baseline

12 weeks

24 weeks

52 weeks

Convensional Biologic

Figure 1

NSAID use in treatment groups during follow-up

\section{Supplementary Files}

This is a list of supplementary files associated with this preprint. Click to download.

- NSAIDsuplementaryfile.docx 